\title{
Production of tENDO1 in stably transformed tobacco cell cultures for mismatch detection.
}

\begin{abstract}
Scanning DNA sequences for polymorphisms and mutations often involve the mismatch specific cleavage by endonucleases at the mismatch sites and subsequent analysis of the digested product for mutation discovery. One of the limitations of using enzymatic mutation detection methods are the cost and availability of a mismatch specific endonuclease. We report the establishment of Nicotiana tabacum L. Cv. Bright Yellow 2 cells stably expressing the truncated ENDO1 (tENDO1) mismatch specific endonuclease. The 5'-Untranslated region of $\mathrm{N}$. tabacum alcohol dehydrogenase gene (NtADH 5'-UTR) under the control of cauliflower mosaic virus (CaMV 35S) promoter was employed to improve the tENDO1 protein yield. To ease the purification process, tENDO1 was secreted into the culture medium and isolated using nickel affinity chromatography. The tENDO1 was estimated to be stably produced in an average of $0.7-0.9 \%$ total soluble protein. Functional test on tENDO1 for mismatch detection demonstrated that tENDO1 retained mismatch specific endonuclease activity resembles its native protein. Further biochemical analysis showed that tENDO1 exhibited mismatch detection specificity and efficiency comparable to other commonly used endonucleases.
\end{abstract}

Keyword: Mismatch detection; Plant cell culture; Secretion; tENDO1; TILLING. 